\title{
AN $\kappa_{0}$-CATEGORICAL THEORY WHOSE LANGUAGE IS COUNTABLY INFINITE
}

\section{ROHIT PARIKH}

ABSTRACT. In the following, we describe an $\boldsymbol{\aleph}_{0}$-categorical, complete, decidable theory $T$ whose language is countably infinite.

$T$ is the theory of a structure $\mathscr{U}$; but before defining $\mathcal{U}$, we define another structure $\mathfrak{U}_{0}$.

Definition. Let $X$ be any set, $\mathcal{F}$ a family of finite subsets of $X$. Then $\mathcal{F}$ is monotone on $X$ if $Y \in \mathcal{F}$ and $Z \subseteq Y$ imply $Z \in \mathcal{F}$. (In algebraic topology, $X, \mathcal{F}$ would be an abstract complex.)

Note that if $\mathcal{F}$ is monotone on $X$ and $X_{1}$ is a finite subset of $X$ then $\mathcal{F}$ restricts in a natural way to a monotone family $\mathcal{F}_{1}$ of finite subsets of $X_{1}$. We shall write $\mathcal{F}_{1}=\mathcal{F} \vdash X_{1}$.

The structure $\mathfrak{Q U}_{0}$ consists of a monotone family $\mathcal{F}$ on $N$ which has the following universality property: Let $Y_{1}, Y_{2}, \mathcal{F}_{1}, \mathcal{F}_{2}$ be such that $Y_{1} \subseteq Y_{2}$, $Y_{2}$ is finite, $\mathcal{F}_{2}$ is monotone on $Y_{2}$ and $\mathcal{F}_{1}=\mathcal{F}_{2}\left\lceil Y_{1}\right.$. Then given an isomorphism $\phi$ of $Y_{1}, \mathcal{F}_{1}$ into $N, \mathcal{F}, \phi$ can be extended to an isomorphism of

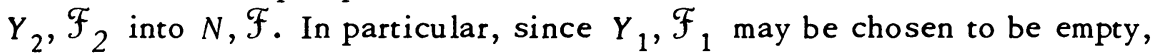
$N, \mathcal{F}$ contains a copy of an arbitrarily chosen $Y_{2}, \mathcal{F}_{2}$.

We show how to construct $\mathcal{F}$. In the above, we can assume without loss of generality that $Y_{1} \subseteq N, \mathcal{F}_{1}=\mathcal{F} \uparrow Y_{1}$ and $Y_{1} \subseteq Y_{2} \subseteq N$, but $\mathcal{F}_{2}$ of course need not be $\mathcal{F} \vdash Y_{2}$.

Enumerate all triples $\left\langle Y_{1}, Y_{2}, \mathcal{F}_{2}\right\rangle$ where $Y_{1} \subseteq Y_{2} \subseteq N, Y_{2}$ is finite and $\mathcal{F}_{2}$ is monotone on $Y_{2}$. We shall take $\mathcal{F}_{1}$ to be $\mathcal{F}_{2} \uparrow Y_{1}$. We assume that each triple occurs infinitely often in our enumeration.

At stage 0 , we let $X_{0}=\varnothing, \varrho_{0}=\varnothing$.

Suppose at stage $n$ we have defined $X_{n}$, a finite subset of $N$, and $\bigodot_{n}$, a monotone family on $X_{n}$. We look at the $n$th triple $t_{n}=\left\langle Y_{1}, Y_{2}, \mathcal{F}_{2}\right\rangle$. We take $X_{n+1}=X_{n}$ and $\varrho_{n+1}=\varrho_{n}$ unless $Y_{1} \subseteq X_{n}$ and $\mathcal{F}_{1}=\varrho_{n}\left\lceil Y_{1}\right.$. If these two conditions do hold, then we let $p=\max \left(X_{n}\right)+1$. Let $Y_{2}^{\prime}=Y_{1} \cup$ $\left\{\left(Y_{2}-Y_{1}\right)+p\right\} . \mathcal{F}_{2}$ immediately converts to an isomorphic monotone family

Presented to the Society, September 24, 1973 under the title $\boldsymbol{\gamma}_{0}$-categorical theories; received by the editors February 8, 1974.

AMS (MOS) subject classifications (1970). Primary $02 \mathrm{H} 13$. 
$\mathcal{F}_{2}^{\prime}$ on $Y_{2}^{\prime}$. We now take $X_{n+1}=X_{n} \cup Y_{2}^{\prime}, \biguplus_{n+1}=\biguplus_{n} \cup \mathcal{F}_{2}^{\prime}$.

The construction ensures that $\bigcup X_{n}=N$. We take $\mathcal{F}=\bigcup_{\mathcal{S}_{n}}$; then $\mathcal{F}$ has the required properties. We shall say that $X \subseteq N$ is $\operatorname{good}$ if $X \in \mathcal{F}$. $\mathcal{U}_{0}$ is $\langle N, \mathcal{F}\rangle$.

The structure $\mathcal{U}$ has base set $N$ and one $n$-ary relation $R_{n}$ for each $n \geq 1 . R_{n}\left(a_{1}, \cdots, a_{n}\right)$ iff the $a_{i}$ are all distinct and $\left\{a_{1}, \cdots, a_{n}\right\}$ is good. $T=\operatorname{Th}(\mathcal{U})$.

Theorem. The theory $T$ described above is $\boldsymbol{N}_{0}$-categorical, complete and decidable; and $R_{n+1}$ is not definable in $T$ from $R_{1}, \cdots, R_{n}$.

Proof. Consider a theory $T_{1}$ whose language is also $L(T)$ and whose axioms are:

(1) Axioms saying that $R_{n}\left(a_{1}, \cdots, a_{n}\right)$ implies that all the $a_{i}$ are distinct.

(2) Axioms saying that $R_{n}\left(a_{1}, \cdots, a_{n}\right)$ and $\left\{b_{1}, \cdots, b_{m}\right\} \subseteq\left\{a_{1}, \cdots\right.$, $\left.a_{n}\right\}$ imply $R_{m}\left(b_{1}, \cdots, b_{m}\right)$ provided only that the $b_{i}$ are all distinct.

(3) For each $X, Y, \mathcal{F}_{1}, \mathcal{F}_{2}$, where $\mathcal{F}_{2}$ is monotone on $Y, X \subseteq Y, \mathcal{F}_{1}=$ $\mathcal{F}_{2} \uparrow X, X=\left\{x_{1}, \cdots, x_{n}\right\}$ and $Y=\left\{x_{1}, \cdots, x_{n}, y_{1}, \cdots, y_{p}\right\}$, the axiom that says that for all $x_{1}, \cdots, x_{n}$, if $x_{1}, \cdots, x_{n}$ "'satisfy" ' $\mathcal{F}_{1}$, then there exist $y_{1}, \cdots, y_{p}$ such that $x_{1}, \cdots, x_{n}, y_{1}, \cdots, y_{p}$ satisfy $\mathscr{F}_{2}$.

All these axioms can be expressed in $L(T)$.

The following are immediate.

(a) $T_{1}$ only has infinite models.

(b) Every countable model of $T_{1}$ is isomorphic to 21 .

Statement (b) can be proved by a "back and forth" argument, building up the isomorphism on finite subsets of the two models in question. The argument is very like that used in showing that the theory of dense linear order without first or last element is $\boldsymbol{N}_{0}$-categorical.

Thus $T_{1}=T$ and $T$ is decidable, complete, $\kappa_{0}$-categorical. To see the last part of the theorem, we prove the following by induction on the complexity of $A$ and axioms of type (3) above:

Let $A\left(x_{1}, \cdots, x_{p}\right)$ be a formula containing only predicate symbols $R_{i}$ with $i \leq n$. Let $\alpha_{1}, \cdots, \alpha_{p} ; \beta_{1}, \cdots, \beta_{p}$ be natural numbers (elements of $\mid$ U| $)$ such that for $e \leq n, R_{e}\left(\alpha_{j_{1}}, \cdots, \alpha_{j_{e}}\right)$ iff $R_{e}\left(\beta_{j_{1}}, \cdots, \beta_{j_{e}}\right)$. Then U $\vDash A\left(\alpha_{1}, \cdots, \alpha_{p}\right)$ iff $\mathcal{U} \models A\left(\beta_{1}, \cdots, \beta_{p}\right)$.

Suppose now that $p=n+1,\left\{\alpha_{1}, \cdots, \alpha_{p}\right\}$ is good, i.e. $R_{n+1}\left(\alpha_{1}, \cdots, \alpha_{p}\right)$ holds. On the other hand $\left\{\beta_{1}, \cdots, \beta_{p}\right\}$ is not good but every proper subset is. Then for a formula $A$ as above, U $\vDash A\left(\alpha_{1}, \cdots, \alpha_{p}\right)$ iff \& $\models$ 
$A\left(\beta_{1}, \cdots, \beta_{p}\right)$. However, $R_{n+1}\left(\alpha_{1}, \cdots, \alpha_{p}\right)$ holds and $R_{n+1}\left(\beta_{1}, \cdots, \beta_{p}\right)$ does not. Thus $R_{n+1}$ is not definable from $R_{1}, \cdots, R_{n}$. Q.E.D.

Suppose now that we have a finite set $F$ of notions definable in $T$. Then they will be definable from some finite set $R_{1}, \cdots, R_{n}$. But then $R_{n+1}$ is not definable from $F$. Hence the language of $T$ is essentially infinite.

\section{REF ERENCE}

1. J. R. Shoenfield, Mathematical logic, Addison-Wesley, Reading, Mass., 1967. MR 37 \#1224.

DEPARTMENT OF MATHEMATICS, BOSTON UNIVERSITY, BOSTON, MASSACHUSETTS 02215 